\title{
Atypical Manifestations of Oral Candidiasis Mimicking Lichen Planus
}

\author{
Lilly Esquivel-Pedraza ${ }^{1,2 *}$, Laura Fernández-Cuevas ${ }^{3}$, Alba Cicero-Casarrubias ${ }^{1}$, Judith Domínguez- \\ Chérit ${ }^{1}$, Silvia Méndez-Flores ${ }^{1}$ \\ ${ }^{T}$ Dermatology Department, National Institute of Medical Science and Nutrition "Salvador Zubirán", Mexico City, \\ Mexico \\ ${ }^{2}$ Health Care Department, Metropolitan Autonomous University, Mexico City, Mexico \\ ${ }^{3}$ Oral Pathology \& Medicine Clinic, Dermatology Center “Dr. Ladislao de la Pascua”, Mexico City, Mexico
}

\section{*Corresponding Author}

Lilly Esquivel-Pedraza

\section{Article History}

Received: 08.07.2020

Accepted: 16.07 .2020

Published: 21.07.2020

\begin{abstract}
Background: Oral candidal infections are predominantly caused by Candida albicans. There are multiple clinical presentations of oral candidiasis. Main observations: We describe two cases of atypical clinical manifestations of oral candidiasis. Patients presented with white reticular plaques, painful ulcers, erosions, and significant oral mucosa atrophy mimicking oral lichen planus. Histopathological findings are detailed. Conclusions: To our knowledge, this is a novel clinical presentation of oral candidiasis, and we propose to consider this variant, particularly in patients with predisposition to develop chronic oral candidiasis.
\end{abstract}

Keywords: Mouth diseases, candidiasis, lichen planus.

\section{INTRODUCTION}

Oral candidal infections are predominantly caused by Candida albicans and many clinical forms have been described [1, 2]. Pseudomembranous candidiasis, also known as thrush, clinically presents as confluent white plaques resembling curdled milk and is usually asymptomatic. Superficially, the plaques can be scraped and the underlying mucosa often exhibits an erythematous appearance [3, 4]. Chronic mucocutaneous candidiasis is a heterogeneous disorder often characterized by the involvement of mucosa, skin and nails $[4,5]$. Erythematous candidiasis clinically presents as localized erythema of the oral mucosa with or without associated symptoms [4, 6]; Denture stomatitis is a variant of erythematous candidiasis, characterized by inflamed mucosa under the prosthetic denture [7, 8]. Median rhomboid glossitis, also known as central papillary atrophy, is another variant of erythematous candidiasis [9]; it presents as an erythematous, well-demarcated, rhomboid or elliptical area of atrophy of the papillae of the dorsal tongue. Hyperplastic candidiasis appears as a white plaque that is non-scrapable, and often mimics leukoplakia [10]; it is usually located to the anterior buccal mucosa and is commonly well demarcated, slightly elevated and adherent [6]. It can appear as small translucent white lesions or as large, dense opaque plaques. It can also present either as an isolated, adherent white plaque (homogeneous type) or as multiple white nodules on an erythematous background (nodular or speckled type) [10].

Other oral conditions are related to candidal infection, such as angular cheilitis, linear gingival erythema and inflammatory papillary hyperplasia. Angular cheilitis-associated candidiasis is a chronic inflammatory lesion characterized clinically by erythema, maceration, crusting, and fissures affecting the lip commissures [3]. Linear gingival erythema manifests as a millimetric linear erythematous band on the marginal gingiva, and is typically observed in HIVpatients [11]. Inflammatory papillary hyperplasia is a tissue-reactive overgrowth characterized by hyperemic mucosa with nodular or papillary appearance in the palate, it is strongly related to C. albicans and the use of dental prostheses [12].

Copyright @ 2020: This is an open-access article distributed under the terms of the Creative Commons Attribution license which permits unrestricted use, distribution, and reproduction in any medium for non commercial use (NonCommercial, or CC-BY-NC) provided the original author and source are credited. 
Oral candidiasis presenting as white reticular and erosive plaques has not been previously described; therefore, the aim of this manuscript is to detail this atypical manifestation in two patients and to analyze the possible role of the mycosis in the oral mucosa lichenification.

\section{Case Report}

\section{Patient 1}

A 20 year-old female patient with a previous diagnosis of primary immunodeficiency and history of multiple bacterial skin infections on the abdominal wall and proximal lower limbs that required antibiotic treatment and occasional surgical approach due to multiple abscess formation, presented with chronic oral ulcers. The patient also had history of recurrent oral candidiasis since she was three months old.

The oral lesions were previously diagnosed as recurrent aphthous ulcers by an odontologist and were treated with antimicrobial oral rinses, antibiotics and topical corticosteroids without improvement and the subsequent development of thrush treated with topical miconazole. On oral exam, the patient presented angular cheilitis, commissural scars, painful white reticular, non-wipeable, bilateral plaques, erosions, and superficial ulcers in buccal mucosa; tongue atrophy and erythema on palate, xerostomia and hypermelanosis (Figure 1 A-C), these lesions had been present for several months. We took biopsies from the buccal mucosa and dorsum of the tongue with an initial clinical diagnoses of lichen planus and erythematosus candidiasis, as well as nutritional deficiency. We indicated the suspension of all the previous treatments.

Histopathological analysis revealed epithelial atrophy with thickening of the basal membrane and an intense lichenoid lymphocityc infiltrate (Figure $1 \mathrm{E}$ ); the inflammatory cells extended deeply into the submucosa involving muscular fibers. Fungal structures (hyphae) were detected in the oral epithelium (Figure $1 \mathrm{~F} \& \mathrm{G}$ ), confirming the clinical diagnosis of candidiasis while ruling-out lichen planus. Blood analyses including vitamin $\mathrm{B}_{12}$ and folic acid, were all within normal ranges.

After one week without drugs, white removable plaques typical of pseudomembranous candidiasis developed; the patient was restarted on antifungals (nystatin rinses and systemic fluconazole (50 $\mathrm{mg}$ weekly/one month) with subsequent resolution of the oral lesions (Figure-1D).

After twelve years of follow-up, the patient has presented multiple recurrences of erythematous candidiasis, mainly associated to antibiotic use. She has been treated with topical nystatin and miconazole. She has not presented reticular plaques or ulcers again.

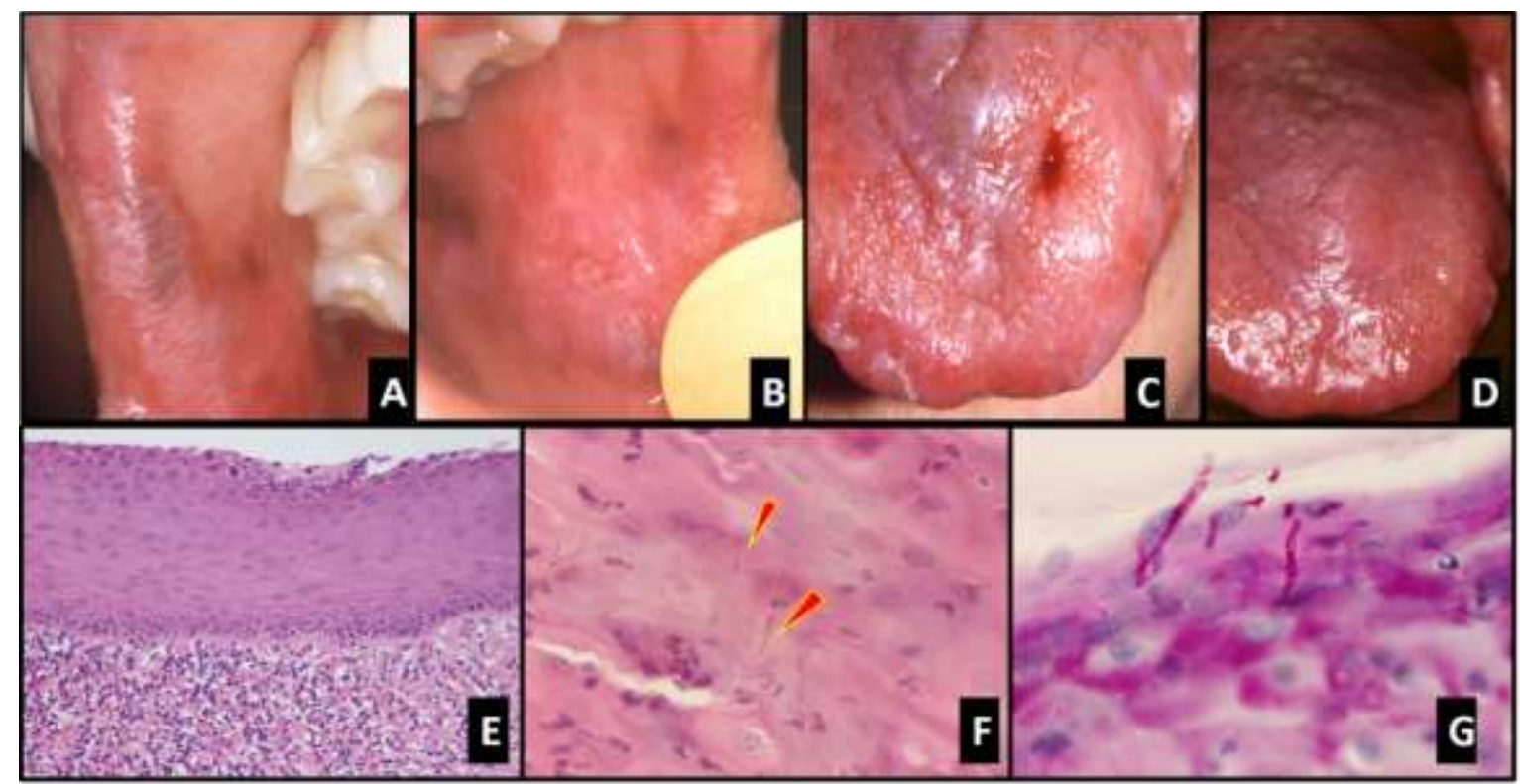

Fig-1: A) White non-scrapable reticular plaques with surrounding erythema and postinflammatory hyperpigmentation in the right retrocomissural mucosa. B) White reticular plaque with erosive margins and millimetric superficial central ulceration on the left buccal mucosa. C. Generalized atrophy of the filiform and fungiform papillae on the dorsum of the tongue, and white non-scrapable reticular plaques with a scarred appearance on the tip of the tongue. In the center, the site where the punch biopsy was taken is observed. D) Clinical image of the dorsum of the tongue after antifungal treatment. E) Histopathological image shows an intense subepithelial lichenoid infiltrate. Note the thickening of the basal interface (H\&E stain, 10x). F) A close-up view shows the presence of hyphae (arrow heads) in the whole thickness of the oral epithelium. (H\&E stain, 40x). G) Periodic acid-Schiff stain emphasizes the presence of Candida sp hyphae in the stratum corneum (40x) 


\section{Patient 2}

A 51 year-old female patient was referred to our Oral pathology office in order to rule out the diagnosis of oral lupus erythematosus. The patient was a smoker, and had used antibiotics as treatment for cystitis and psychotropics for insomnia. Also, she had a previous clinical and microscopic diagnosis of oral lichen planus, and had received thalidomide, non-steroidal anti-inflammatory drugs (NSAIDs), corticosteroids, retinoids, and tacrolimus, without improvement.

On her first visit, we observed painful chronic bilateral erosive, reticular, non wipeable, white patches, located on the lateral borders of the tongue, buccal and labial mucosa associated to xerostomia and generalized erythema (Figure 2 A-C). The above findings were suggestive of erythematous candidiasis and erosive lichen planus. However, after revision of slides, candidal infection was confirmed but lichen planus diagnosis was discarded microscopically. The oral mucosa biopsy specimen (Figure $2 \mathrm{E} \& \mathrm{~F}$ ) demonstrated a lichenoid inflammatory infiltrate of lymphocytes with vacuolar alteration of basal keratinocytes, and an eosinophilic band beneath the basement membrane. High power view showed hyphal forms suggestive of Candida overlying oral epithelium. Periodic acid-Schiff stain was positive to candidal hyphae. The patient presented resolution of the oral lesions and symptoms after antifungal treatment with topical clotrimazole (200 mg twice daily $/ 4$ weeks), and systemic fluconazole (100 mg weekly/one month) (Figure 2D).

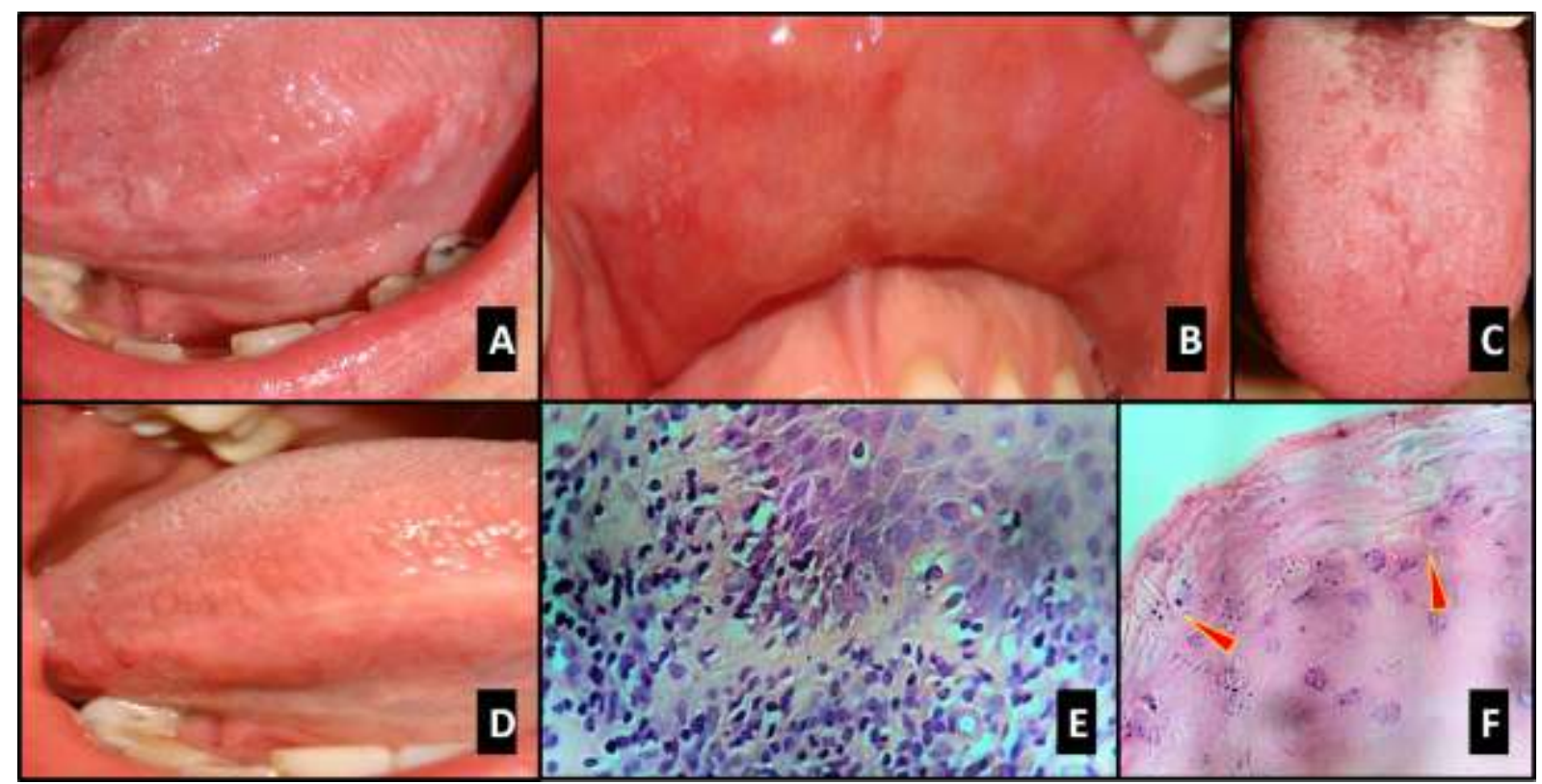

Fig-2: A) Non-wipeable white erosive reticular plaque on the left lateral border of the tongue. B) Subtle bilateral non-wipeable white reticular and speckled plaques with surrounding erythema and punctiform ulcers on the upper labial mucosa. C) Erythematous candidiasis in the middle third of the tongue, contrasting to the contiguous thick and homogeneous white furring plaque on the dorsum of the tongue. D) Clinical resolution of lateral border of the tongue lesions after antifungal treatment. E) Microscopic lichenoid pattern (H\&E stain, 40x). F) Hyphae (arrow heads) in the stratum corneum (H\&E stain, 40x)

\section{DISCUSSION}

The diagnosis of oral candidiasis is often based on clinical examination and thorough history. Additional adjunctive diagnostic methods such as direct examination of smears, culture and biopsy are valuable in confirming the diagnosis $[4,13]$. Biopsy is usually performed for the diagnosis of hyperplastic candidiasis, thus it has been suggested that this condition probably represents candidiasis superimposed on a preexisting leukoplakia [14]. Some believe that Candida alone is capable of inducing hyperkeratotic lesions [4]. In our patients the presence of candidiasis was confirmed through histopathological analysis (Figs $1 \mathrm{~F}, \mathrm{G} \& 2 \mathrm{~F}$ ), in both cases a lichenoid pattern was observed, and according to current diagnostic criteria, lichen planus was ruled-out [15]. The atypical picture in both patients could have been secondary to the chronicity of the candidal infection and the associated use of corticosteroids, resulting in bilateral erosive and reticular plaques mimicking lichen planus clinically. This latter presentation differed substantially from the homogeneous (smooth), or speckled plaque types known in hyperplastic candidiasis. In both cases, the presence of mycotic infection was confirmed histologically from the non-scrapable bilateral white reticular plaques that simulated lichen planus. Both cases presented clinical resolution of the lesions after antifungal treatment, and did not respond to conventional (steroids) treatment to oral lichen planus. These cases underscore the capacity of this fungus to form a reticular white pattern on the oral mucosa associated to an intense lichenoid infiltrate in the submucosa that provokes erosions and ulcers simulating lichen planus, but with improvement with antifungal treatment instead of topical corticosteroids $[15,16]$. 
Superficial fungal diseases can lead to significant morbidity and potentially fatal outcome [17]. Oral candidiasis is usually asymptomatic; however, it can cause oral discomfort, mild soreness, burning sensation, pain, dysguesia, and aversion to food [4, 18], mainly when accompanied by a loss of filiform papillae on the dorsum of the tongue [4]. It has been well established that the presence of chronic candidiasis may manifest as mucosal atrophy [19]. Also, it has been described that fissures and tongue atrophy are risk factors for development of oral candidiasis [20]. On the other hand, it has been postulated that tongue atrophy is mainly observed in nutritional deficiencies [21] and that candidiasis may have its onset after the loss of tongue papillae instead of being the cause of atrophy [14, 19]. Our patients presented morbidity associated with mucosal atrophy, secondary inflammation related to chronic candidiasis and subsequent formation of persistent painful ulcers. In the first case, the presence of lifelong candidiasis secondary to primary immunosuppression, and the absence of nutritional deficiencies, suggest the former favored the development of generalized mucosal atrophy.

Finally, a synchronic relation of oral candidiasis with the presence of leukoplakia, lichen planus, lupus erythematosus, or HIV infection has been widely observed [4, 22-24]. Nevertheless, lichenification of the oral mucosa secondary to candida infection is a scarcely studied phenomenon in the literature. The clinicopathological manifestations of both of the cases presented suggest the possibility of a previously non-reported clinical subtype of oral candidiasis; we propose it should be considered as an unusual clinical variant in patients with predisposition to develop chronic candidiasis, like the immunocompromise and long-lasting xerostomia observed in our patients. We encourage further research to analyze the role of chronic candidiasis on the oral mucosa favoring reticular patterns.

\section{CONClusions}

In summary, the cases reported in this article lack the classic clinical characteristics previously reported in oral candidiasis. It is possible that among others, the conjunction of chronic candidal infection, xerostomia, and systemic/local immunosuppression in our patients favored this clinical picture. Based on these cases, it is advisable to consider oral candidiasis in the differential diagnosis of white non-scrapable reticular plaques, particularly in patients with risk factors for chronic candida infection.

\section{ACKNOWLEDGMENTS}

All authors would like to thank Yosseline Merari Sánchez-Jiménez, DDS, Zaira Paola Valencia Gutiérrez and Jessica Pérez-Olivera dietitians for their technical assistance.

\section{Financial Disclosure and Research Funding: None}

\section{Conflict of Interest: None reported}

\section{REFERENCES}

1. Manfredi M, Polonelli L, Aguirre Urizar JM, Carrozzo M, McCullough MJ. Urban legends series: oral candidosis. Oral diseases, 19(3):245-61.

2. Singh, A., Verma, R., Murari, A., \& Agrawal, A. (2014). Oral candidiasis: An overview. Journal of oral and maxillofacial pathology: JOMFP, 18(Suppl 1), S81.

3. Reichart, P. A., Samaranayake, L. P., \& Philipsen, H. P. (2000). Pathology and clinical correlates in oral candidiasis and its variants: a review. Oral diseases, 6(2), 85-91.

4. Krishnan, P. A. (2012). Fungal infections of the oral mucosa. Indian journal of dental research, 23(5), 650-659.

5. Julián-González, R. E., Valdebrán-Canales, M. A., \& Guidos-Morales, H. E. (2010). Chronic mucocutaneous candidiasis: Case report. Archivos argentinos de pediatria, 108(2), e37-40.

6. Farah, C. S., Ashman, R. B., \& Challacombe, S. J. (2000). Oral candidosis. Clinics in dermatology, 18(5), 553-562.

7. Ramage, G., Tomsett, K., Wickes, B. L., López-Ribot, J. L., \& Redding, S. W. (2004). Denture stomatitis: a role for Candida biofilms. Oral Surgery, Oral Medicine, Oral Pathology, Oral Radiology, and Endodontology, 98(1), 5359.

8. Araújo de Vasconcellos, A., Araújo de Vasconcellos, A., Chagas, R. B., \& Gonçalves, L. M. (2014). CandidaAssociated Denture Stomatitis: Clinical Relevant Aspects. Clin Microbial. 3: 160.

9. Nelson, B. L., \& Thompson, L. D. (2007). Median rhomboid glossitis. Ear, Nose \& Throat Journal, 86(10), 600601.

10. Sitheeque, M. A. M., \& Samaranayake, L. P. (2003). Chronic hyperplastic candidosis/candidiasis (candidal leukoplakia). Critical Reviews in Oral Biology \& Medicine, 14(4), 253-267.

11. Patil, S., Rao, R. S., Majumdar, B., \& Anil, S. (2015). Clinical appearance of oral Candida infection and therapeutic strategies. Frontiers in microbiology, 6, 1391.

12. Goulart, M. C. V., \& Lara, V. S. (2011). Inflammatory papillary hyperplasia of the palate: quantitative analysis of candida albicans and its negative correlation with microscopic and demographic aspects. International Journal of 
Prosthodontics, 24(3):235-237.

13. Giannini, P. J., \& Shetty, K. V. (2011). Diagnosis and management of oral candidiasis. Otolaryngologic Clinics of North America, 44(1), 231-240.

14. Scully, C., Ei-Kabir, M., \& Samaranayake, L. P. (1994). Candida and oral candidosis: a review. Critical Reviews in Oral Biology \& Medicine, 5(2), 125-157.

15. Van der Meij, E. H., \& Van der Waal, I. (2003). Lack of clinicopathologic correlation in the diagnosis of oral lichen planus based on the presently available diagnostic criteria and suggestions for modifications. Journal of oral pathology \& medicine, 32(9), 507-512.

16. Juneja, M., Mahajan, S., Rao, N. N., George, T., \& Boaz, K. (2006). Histochemical analysis of pathological alterations in oral lichen planus and oral lichenoid lesions. Journal of oral science, 48(4), 185-193.

17. Lanternier, F., Cypowyj, S., Picard, C., Bustamante, J., Lortholary, O., Casanova, J. L., \& Puel, A. (2013). Primary immunodeficiencies underlying fungal infections. Current opinion in pediatrics, 25(6), 736-747.

18. Samaranayake, L. P., Leung, W. K., \& Jin, L. (2009). Oral mucosal fungal infections. Periodontology 2000, 49(1), 39-59.

19. Terai, H., \& Shimahara, M. (2005). Atrophic tongue associated with Candida. Journal of oral pathology \& medicine, 34(7), 397-400.

20. Miyashita, M., Baba, S., \& Suzuki, H. (1995). Role of recurrent oral candidiasis associated with lingua plicata in the Melkersson- Rosenthal syndrome. British Journal of Dermatology, 132(2), 311-312.

21. Cunha, S. F. D. C. D., Melo, D. A. D., Braga, C. B. M., Vannucchi, H., \& Cunha, D. F. D. (2012). Papillary atrophy of the tongue and nutritional status of hospitalized alcoholics. Anais brasileiros de dermatologia, 87(1), 84-89.

22. Arora, S., Verma, M., Gupta, S. R., Urs, A. B., Dhakad, M. S., \& Kaur, R. (2016). Phenotypic variability and therapeutic implications of Candida species in patients with oral lichen planus. Biotechnic \& Histochemistry, 91(4), 237-241.

23. Krogh, P., Holmstrup, P., Thorn, J. J., Vedtofte, P., \& Pindborg, J. J. (1987). Yeast species and biotypes associated with oral leukoplakia and lichen planus. Oral surgery, oral medicine, oral pathology, 63(1), 48-54.

24. Fangtham, M., Magder, L. S., \& Petri, M. A. (2014). Oral candidiasis in systemic lupus erythematosus. Lupus, 23(7), 684-690. 